\title{
ORGANIZACIÓN COMUNITARIA Y CIUDADANÍA INDÍGENA EN EL MUNICIPIO DE IXMIQUILPAN, HIDALGO
}

\section{COMMUNITY ORGANIZATION AND INDIGENOUS CITIZENSHIP IN THE MUNICIPALITY OF IXMIQUILPAN, HIDALGO}

\author{
(iD) Alan Suah Islas Ruiz ${ }^{1^{*}}$ \\ mpdr0419@elcolegiodehidalgo.edu.mx \\ ${ }^{1}$ El Colegio del Estado de Hidalgo, México
}

\begin{abstract}
*Correspondencia: Alan Suah Islas Ruiz. Email: mpdr0419@elcolegiodehidalgo.edu.mx
\end{abstract}
Recibido: 16.07.21 | Aprobado: 16.08.21

\section{RESUMEN}

Uno de los retos en la agenda de los asuntos indígenas ha sido el vínculo entre el sistema político-jurídico del Estado, y los mecanismos de organización y participación del sistema de usos y costumbres que busca reconocer la diversidad cultural indígena. Dicha relación refleja el tratamiento del Estado hacia las ciudadanías que proponen las organizaciones políticas indígenas como en el caso de Ixmiquilpan, en el estado de Hidalgo, México. En este texto se abordan algunas cuestiones para entender cómo ha sido este vínculo entre las organizaciones comunitarias indígenas y el sistema político, por medio del análisis de su estructura social y política, y los mecanismos de participación derivados de ella. Se parte del institucionalismo histórico como herramienta teórica para explicar cómo las estructuras políticas de usos y costumbres han ido formando un andamiaje jurídico-político no necesariamente compatible con las instituciones políticas que plantea el Estado mexicano.

Palabras clave: Organización comunitaria, ciudadanía indígena, usos y costumbres, Ixmiquilpan Hidalgo.

\begin{abstract}
One of the main challenges on the agenda of indigenous affairs has been related to the link between the political-legal system and the mechanisms recognized by the system of uses and customs to integrate variety in political participation, and the community organization, mainly of rural contexts. Said relationship reflects the State's treatment of difference, multiculturalism, and citizenships proposed by an indigenous political organization of customary laws, as in the case of the municipality of Ixmiquilpan, in the state of Hidalgo, Mexico. This article addresses some questions to understand how this link has been between indigenous society and the political system, through the analysis of its community organization: its social and political structure, and the mechanisms of citizen participation derived from it. It starts from historical institutionalism as a theoretical tool to explain how the political structures of customs and uses have been forming a legal-political scaffolding that is not necessarily compatible with the political institutions proposed by the Mexican State.
\end{abstract}

Keywords: Community organization, indigenous citizenship, customary laws, Ixmiquilpan Hidalgo. 


\section{INTRODUCCIÓN}

La pugna por el reconocimiento de la autonomía indígena y la reforma al Art. 2 Constitucional tuvo como una de sus bases más relevantes el movimiento zapatista de 1994 (Arias, 2005). Con la firma de los tratados de San Andrés en 1996, se ponía de cierta forma fin al monopolio de los partidos y asociaciones obrero-campesinas que históricamente habían encauzado la participación de los grupos indígenas en los procesos político-electorales. El ejercicio de la ciudadanía de contextos indígenas estaba acotado por este tipo de organizaciones (partidos, congregaciones obreras, etc.). De cierta forma se había configurado con este tratamiento un margen de control político hacia lo indígena, que para los gobiernos en turno representaban meramente grandes reservas electorales, quienes a cambio recibían principalmente apoyos de programas sociales. Esta forma de entender cómo se vinculaba el Estado respecto de los grupos indígenas puede entenderse como una ciudadanía social cuyo marco de referencia promovía una especie de Estado de bienestar (Tamayo, 2006).

Hidalgo es uno de los estados de México con mayor historia política indígena, no sólo por la cantidad de hablantes, sino por la importancia de sus organizaciones, movimientos e instituciones en la vida política del Estado. Con la crisis económica iniciada en lo que se conoce como la "década perdida", la política hacia el sector indígena cambió para buscar integrarlos a la dinámica de industrialización y privatización que permeaba en la vida pública. Este discurso, empero, necesitaba legitimarse dentro de las propias organizaciones políticas del Estado. Esto resultó en la posterior aparición de instituciones y programas específicos para apoyar al sector indígena desde los noventa hasta la actualidad.

La estructura del documento comienza por discutir a partir del institucionalismo histórico el origen del tratamiento de lo indígena en la época Cardenista, y su relación con la creación en la región del Patrimonio Indígena del Valle del Mezquital (PIVM). Posteriormente, se habla acerca de cómo la estructura social que se fragua con la organización de usos y costumbres en el municipio de Ixmiquilpan, Hidalgo, deriva en la construcción de una identidad indígena, y cómo desde esta se configuran espacios para participar y organizarse, es decir, cómo surge en el escenario indígena una ciudadanía con características particulares que no necesariamente se compaginan con el proyecto políticojurídico de la ciudadanía liberal universalista que plantea el Estado a través de sus instituciones y organismos autónomos como el Instituto Nacional Electoral (INE).

\section{MARCO TEÓRICO-METODOLÓGICO}

El control político de lo indígena. Un acercamiento desde el institucionalismo histórico

Luego de que se agotaran las explicaciones del institucionalismo clásico acerca del comportamiento de los sujetos en las organizaciones, se pensó en la pertinencia de recuperar algunos de los postulados fundamentales de las ciencias sociales para comprender el cambio social y político de la época de posguerra. La subsecuente transición hacia la democracia y el auge de movimientos sociales importantes en América Latina, por ejemplo, llevó a las y los investigadores a repensar el papel de la historia para indagar la forma en que se llevan a cabo las acciones sociales, principalmente en contraste 
con las perspectivas racionalistas y conductistas, que hasta ese entonces habían hegemonizado el corpus teórico de la política y los procesos políticos dentro de las sociedades (O’Donnell, 2000; Mainwaring y Welna, 2003).

El institucionalismo histórico plantea una revisión crítica en la formulación de explicaciones causales en el tiempo, pues ante la complejidad de información respecto de un fenómeno social, se construye un marco explicativo que selecciona los eventos con mayor importancia para el desarrollo de los comportamientos sociales. Como característica fundamental, Pierson y Skocpol (2008) arguyen que este tipo de estudios se fundamentan en el interés por la amplitud en su abordaje, y al mismo tiempo por lo sustantivo en la conexión de las variables que explican al fenómeno en cuestión. Por tanto, se abre la posibilidad de refutar planteamientos aparentemente universalistas o particularistas.

En ese sentido, se distingue de otros enfoques neoinstitucionalistas como la noción del cálculo instrumentalista del comportamiento. Desde esa perspectiva, las instituciones son esquemas que fijan un espacio en el que los individuos se desenvuelven, estableciendo criterios racionales que optimicen los resultados de sus preferencias, símil de la dinámica de interacción que establece el mercado (Coase, 1937; North, 1995; DiMaggio y Powell, 1983).

El enfoque sociológico modificó el centro de discusión del cambio institucional hacia los individuos que las conforman, y el contexto social que influye sus acciones. Las instituciones son entonces construcciones sociales, producto del conflicto y el acuerdo (March y Olsen, 1989; Ostrom, 2000).

El puente epistemológico construido por el institucionalismo histórico es un esfuerzo por entender sistémicamente el cambio social. La coyuntura es un concepto del institucionalismo histórico, el cual refiere justo a la unión entre eventos ligados no necesariamente de forma lineal en el tiempo, sino mediante la importancia de la correlación e interacción dada entre las variables que lo explican (Hernández, 2019).

Es decir, para el institucionalismo histórico importa el establecimiento en primer lugar de una selección de eventos relacionados que son capaces por sí mismos de brindarnos un panorama amplio al estado presente de las organizaciones sociales. Así entonces, la coyuntura que se plantea en este trabajo para el análisis de la organización social indígena se relaciona con el surgimiento de la política Cardenista. Para el estudio se aplicó una metodología cualitativa de corte descriptiva, en la que se usó como técnica de investigación el análisis documental de bibliografía relacionada.

\section{Cardenismo y tratamiento histórico hacia lo indígena}

El autoritario ejercicio del poder político y el exacerbado control social fueron factores que signaron las condiciones del sector indígena, generando cosmovisiones particulares respecto de lo político. Antes de ser Hidalgo, el territorio estuvo compuesto por varios calpixcazgos los cuales se encontraban bajo el dominio español. Estas unidades político-administrativas luego se institucionalizaron como alcaldías, las cuales agudizaron la explotación indígena incluso después de que, con las encomiendas, el sector indígena tuviera ciertos derechos como los servicios médicos (Menes, 2006; Ruiz, 2000). 
Este control histórico sobre los pueblos y comunidades indígenas formó parte de su política y resistencia por defender su cultura originaria, que se remonta a la época prehispánica. Podemos decir que, ante la explotación y despojo de los recursos naturales, los pueblos originarios siempre mantuvieron prácticas de resistencia y conservación de sus costumbres y tradiciones. Ejemplo de ello es la región del Valle del Mezquital, protagonista en la historia de Hidalgo por su rebelión y defensa de sus culturas a lo largo de la historia.

Esta cultura política de resistencia hacia las formas institucionalizadas de poder creó una narrativa de defensa hacia el gobierno. La resistencia hacia el poder en cuanto componente del imaginario colectivo configuró un espacio social donde los conceptos de justicia social, libertad y autonomía encontraron un discurso común, que se fue mezclando con las prácticas del ejercicio del poder político. Dicha combinación no fue problemática porque la desobediencia encontró otros lugares de aparición, en escenarios subculturales o de discursos ocultos (Scott, 2004).

Las comunidades y pueblos indígenas de Ixmiquilpan han fungido como amplias reservas de capital para los grupos políticos de la región. En gran medida, esto tiene que ver con la estructura política que modeló el Partido Revolucionario Institucional (PRI) para con los sectores sociales. El tratamiento corporativista tiene origen en la época Cardenista hacia la primera mitad del siglo XX, donde se fortalecieron los liderazgos de las grandes organizaciones obreras, campesinas e indígenas.

El proceso de aculturación indígena se intensificó con Lázaro Cárdenas en nuestro país. Durante su período se creó el Departamento de Asuntos Indígenas (DAI), el cual era la instancia gubernamental encargada de integrar las cuestiones indígenas al desarrollo del país. Según la propia narrativa de la institución bajo el principio de justicia social (López, 2013). La idea era construir una serie de instrumentos de intervención en el medio indígena para "sacarlos" de alguna forma del "atraso" en el que se encontraban. Incrementó el presupuesto para educación y las oficinas gubernamentales se acercaron a las comunidades indígenas.

Por otra parte, se diseñó una imagen de Cárdenas como líder social para invitar a los grupos indígenas a no desconfiar del gobierno, pues se estaba en el entendido de que los grupos "indios" se encontraban a la defensiva de las invasiones de los grupos "blancos". Evidentemente tal cuestión requería algo más que una eficiente estrategia de comunicación social, para ello se formaron grupos de investigación, los cuales tuvieron como tarea estudiar al sector indígena para comprender su forma de pensar y vivir lo político (López, 2013). De esta forma se lograron aglutinar una serie de organizaciones tanto académicas como sociales para pensar concretamente en los medios disponibles para mejorar el estado de los pueblos indígenas otomíes de la región. El DAI Cardenista fue una dependencia ligada directamente al ejecutivo federal, por lo que a raíz de esta vinculación se propuso que la política indigenista se desconcentrara tanto territorialmente como para los gobiernos subnacionales.

Hacia 1952 se creó entonces el Patrimonio Indígena del Valle del Mezquital (PIVM), el cual era un fideicomiso público que tenía como objeto enviar recursos a esa región para activar su economía y salir del "atraso" en que se encontraba. Los pobladores 
narran ese hecho como una acción que benefició a las comunidades: otorgando trabajos prácticamente a la mayoría de los habitantes de los pueblos del Mezquital (Quezada, 2015). En 1982 se integró al fideicomiso la región de la Huasteca, permitiendo así detonar una serie de condiciones económicas, con base en criterios de integración funcional. El PIVM fue derogado en 1990, resultado de la política de integración basada en el nuevo modelo neoliberal tanto en la economía como en la política del gasto de la administración pública.

La integración de la región Huasteca al PIVM trajo, desde el punto de vista de las políticas públicas, un problema de diseño, pues no consideraba las características propias de la región, y, por el contrario, hacía referencia a un conjunto de condiciones sociales y culturales que el Estado llamó indigenismo.

La política indigenista del PIVM conjuntaba deliberadamente toda la diversidad de cosmovisiones propias de los pueblos y comunidades "originarios". El Patrimonio Indígena del Valle del Mezquital no sólo representaba una propuesta de política económica, sino también era una propuesta de integración política, la cual implicaba o formaba parte del proceso de aculturación Cardenista iniciado casi medio siglo antes.

Tras su desaparición en los noventa, grandes flujos de personas tuvieron que comenzar a emigrar para buscar mejores condiciones de vida, pues sin el PIVM, y sin proyectos de inversión en la región, nuevamente se encontraban en una situación en la que primaba la pobreza y las desigualdades sociales. Paulatinamente, la profundización del modelo neoliberal acrecentó la polarización en los ingresos, al centrarse la política de desarrollo regional en la expansión de los trabajos de los centros urbanos, en tanto cristalización del paradigma de la modernidad y el progreso industrial (Garza, 2003). El naciente ciudadano moderno era quien vivía entonces en las grandes ciudades, y no el que formaba parte de los mundos indígenas.

Puesto que la actividad económica principal de las comunidades indígenas eran las vinculadas con la agricultura, gran parte de la "vida indígena" se desarrollaba en el mundo rural. Después de este crecimiento urbano que empezó desde finales de los setenta, se distingue desde el punto de vista económico, una desruralización del campo, y una ruralización de las ciudades (Méndez, 2005).

A la postre, este proceso de transformación impactó en la organización social dentro de las comunidades y los pueblos indígenas, provocando en la región un creciente flujo migratorio hacia la capital de Hidalgo, Pachuca, donde a partir de los años ochenta surgieron asentamientos periféricos compuestos principalmente por indígenas tanto del Estado como de Estados vecinos (Pérez, 2018).

Ya en las ciudades, las comunidades indígenas -ahora dispersas-, se fueron atomizando e integrando a la cultura urbana que planteaba el modo de vida moderno. Con ese nuevo proceso cultural y político de transición entre lo rural y lo urbano, se comenzaron a generar nuevas concepciones de lo indígena (Ramírez, 2008).

El subsecuente tratamiento del Estado frente a esta realidad fue el planteamiento patrimonialista de lo indígena, donde, desde el punto de las políticas públicas y el accionar gubernamental, se vinculó a lo indígena con el ámbito institucional de la cultura y las artes. Lo indígena debe ser, desde este "segundo patrimonialismo", susceptible de ser protegido 
por el Estado. Entre más original se mantengan las tradiciones indígenas, más valor tienen como productos artísticos dentro del mercado culturalista. Estas nuevas concepciones de lo indígena están ligadas a lo que se conoce como nuevas ruralidades, donde se reconocen nuevas relaciones de lo rural respecto de lo urbano, y dan lugar a espacialidades intersticiales o "rururbanas" (Canabal, 2005).

Derivado de esto, podemos decir que Ixmiquilpan y la región del Valle del Mezquital ha sufrido diversos cambios tanto económicos como políticos relacionados con la dinámica histórica del tratamiento de lo indígena en el ámbito público. Empero, el reconocimiento de su diversidad de iure no ha logrado traducirse en mecanismos que garanticen el cumplimiento de los derechos que establece el propio marco jurídico Constitucional.

Ixmiquilpan es distinguido por su composición mayoritaria de comunidades de usos y costumbres. Por su historia y cultura, la región comparte, además de expresiones culturales comunes, formas comunes de concebir lo político. A esta particularidad en la forma de vincularse con lo político se entiende, desde este punto de vista. como ciudadanías "indígenas" o "étnicas". Dichas ciudadanías van más allá del pensamiento indígena sobre el mundo de lo político, la política y las políticas, pues constituyen prácticas sui generis de participación y expresión política.

\section{DISCUSIÓN}

De las estructuras sociales indígenas a la ciudadanía indígena

Autores como Esposito, consideran a la comunidad como un ente orgánico que se agrega a la naturaleza de los sujetos (2003), de tal modo que éstos, sujetos a una identidad mayor (la comunidad), la busquen preservar en el futuro como esencia de su originalidad presente. Siguiendo a Rentería: “el culto a la originalidad y autenticidad de la cultura aparecen como esencia de las comunidades, pues a partir de ellas se venera al origen mítico (...) y ofrece al imaginario colectivo de los pueblos la cristalización de la sociedad igualitaria" (2016:4).

En ese sentido, Tönnies (1979) explica cómo es posible ver dos grandes tipos de proyectos de socialización en el mundo moderno. Por un lado, el de la comunidad que trata de preservar intereses de grupo tradicionales y, por otro lado, el de la sociedad que se funda en el cálculo racional-individual Occidental.

La democracia puede entenderse como el resultado del segundo proyecto de socialización. Ello porque ha logrado incorporar el pensamiento economicista propio del neoliberalismo que, a su vez, se fundamenta en el individualismo metodológico, según el cual la primacía del comportamiento individual guía los comportamientos colectivos y por tanto los comportamientos ciudadanos. Dicha concepción atraviesa a la misma democracia, pues el agente primario de ésta es el ciudadano; el individuo capaz de racionalizar y tomar decisiones con base en su conocimiento de lo público. Dicho de otra forma; existe una advertida compatibilidad entre el agente central del discurso democrático: el ciudadano, y la base metodológica del neoliberalismo que privilegia la racionalidad individual. 
El discurso de la democracia mantiene entonces diferencias aparentemente contradictorias: el valor de la participación política colectiva como actividad que transforma la política, y el individualismo que funda a la ciudadanía como principal componente de la democracia. La idea de la comunidad se inserta en el primer valor, mientras que rechaza al segundo en cuanto han sido los movimientos sociales indígenas portadores de proyectos alternativos de vida (Velázquez, 2009).

Es decir, la democracia que se empezó a fraguar en nuestro país empezó a tener un cierto matiz que no se acoplaba a las estructuras políticas indígenas tradicionales. La ciudadanización de los organismos del sistema político electoral comenzó a finales de los ochenta, producto de las reformas en materia política que permitían establecer "reglas del juego" más competitivas en el sistema de partidos. La idea de la ciudadanización era poner en el centro de la agenda política una base para continuar con la idea de la formación del ciudadano como el agente de referencia para el cambio y el control político moderno. Es decir, todos los derechos recaerían ya no en las colectividades: en los pueblos ni en las comunidades, -como lo planteaba el proyecto Cardenista-, sino en el individuo capaz de diferenciar entre lo que resulta de interés para sí mismo.

A partir de ello, entendemos a la ciudadanía como una suerte de estatus que consiste en el ejercicio de los derechos políticos inscritos dentro del margen de reconocimiento del Estado. Resulta ser un conjunto de prácticas, actitudes, valoraciones e información específica sobre cómo vincularse con el poder. Es un "paquete" cultural que forma parte del proyecto democrático que promueve el mundo Occidental (Arbona, 2008).

En tal sentido, el discurso de esta nueva ciudadanía ha buscado transformar el estado de la ciudadanía social que en México se caracterizó por el protagonismo de asociaciones campesinas, obreras, y partidos políticos que encabezaban las grandes demandas sociales frente a los gobiernos, incluidas las reservas político-electorales del sector indígena (Tamayo, 2015). En otros términos, la ciudadanía más allá de una delimitación estrictamente dentro del ámbito jurídico es una práctica histórica que se fragua en la cotidianidad, que conlleva entre otras cosas, un constante conflicto entre diferentes posturas sobre cómo vincularse con el Estado y el poder público (López, et al, 2019).

Las reformas políticas que México ha tenido desde la creación del Instituto Federal Electoral a inicios de los años noventa, se basan en el proyecto de sociedad y no el de comunidad. La democracia que ha promovido nuestro país también involucra un modelo de comportamiento individualizado. El antecedente directo es la propuesta de política pública de la Estrategia Nacional de Cultura Cívica del Instituto Nacional Electoral de 2014.

En ese documento se propone, entre otras cosas, generar capacidades para que el ciudadano tenga las herramientas cognitivas y sociales necesarias para participar en la toma de decisiones respetando las reglas del sistema político vigente (INE, 2018). Para los grupos indígenas, la estrategia se torna más ambigua; puesto que busca el respeto y reconocimiento Constitucional a las formas de organización indígena, pero al mismo tiempo los intenta insertar en la dinámica propia de un modelo democrático que hace 
énfasis en el respeto hacia las reglas (sin tener la oportunidad de buscar modificarlas, o al menos ese no es su propósito).

Entre el contraste de estos grandes proyectos: sociedad y comunidad, han aparecido problemas y tensiones relacionados con la apropiación del territorio, la organización política, y la impartición de justicia en materia político-electoral en distintas dimensiones.

La idea del poder-justicia está al fondo de todas, en cuanto es la distribución de las actividades, el trabajo y la organización comunal el medio para la legitimación del sistema de valores, y por tanto la base de la relación que se establece con el Estado (Barkin y Lemus, 2014).

El sistema jurídico de nuestro país, si bien reconoce otras formas de organización y distribución de poder-justicia a través de estructuras políticas indígenas con usos y costumbres, su ejercicio ha tenido variados efectos. Para elegir autoridades con cargos de elección popular, por ejemplo, la Constitución menciona la obligación del Estado para fomentar la participación y representación indígena, empero, se han presentado escasos casos al respecto, además que su aparición ha sido desigual en los sectores indígenas (Singer, 2016). Ello da cuenta del escaso interés o incompatibilidad del proyecto de ciudadanía que se busca implantar, y que dista de la amplia diversidad de las formas de participar en las comunidades indígenas.

La apropiación de la idea de la justicia como principio rector del orden político, por otra parte, no necesariamente atiende a una cuestión de estricta diferenciación entre lo indígena y lo no-indígena. Es decir, la legitimación de las prácticas políticas en las comunidades con usos y costumbres plantean no sólo el problema del reconocimiento a sus estructuras y organizaciones, sino el problema de la coexistencia de diferentes identidades y proyectos en la cultura política de la región (Martínez, 2014).

En Ixmiquilpan la organización política y social funciona a partir de una estructura que "imparte justicia" a través de Asambleas, las cuales tienen el encargo de solucionar los conflictos internos y, además, planear y organizar las diferentes actividades tanto religiosas como de otra clase de tópicos propios de las tradiciones locales (Quezada, 2015).

La Asamblea comunal es el órgano rector de las decisiones, compuesta por ciudadanos hombres y mujeres que eligen al delegado presidente, el cual es la autoridad más importante, y quien funciona como medio de contacto con las autoridades del Ayuntamiento y del Estado. En la estructura también hay un tesorero, subdelegados que representan a cada barrio o colonia, así como un comisariado ejidal que tiene como propósito solventar todos los problemas que tengan que ver con el uso del suelo en la comunidad.

También hay Asambleas que se encuentran compuestas por comités, los cuales son grupos de vecinos que se reúnen para tratar asuntos de temáticas en concreto; de obras públicas, para la organización de las ferias, la administración del panteón, el drenaje, la iglesia, etc. Dichos comités los conforman los vecinos que de una forma u otra tienen injerencia en ese tema, por ejemplo, el comité de las escuelas está conformado por los padres de familia, y el de la iglesia por creyentes "reconocidos". La designación para pertenecer a cada comité corresponde a la Asamblea comunal. 
Es importante decir que, en cada barrio, se realizan faenas, una especie de trabajo comunitario el cual es convocado por el delegado hacia los habitantes (todos participan, con excepción de los adultos mayores, discapacitados, niños y enfermos). En el caso de que alguno de ellos no accediera a la realización de estas faenas, el delegado tiene la facultad para "multar" económicamente a dicha persona. Principalmente las faenas se tratan de labores de limpieza de las calles, drenajes, etc.

El trabajo comunitario es un elemento común en las estructuras de usos y costumbres, donde el ciudadano tiene derecho de participar siempre y cuando contribuya en las actividades comunales. El trabajo comunitario puede adquirir diferentes modalidades. En el país encontramos varias figuras parecidas a las faenas como el tequio o los jornales (Singer, 2016). Estas figuras implican una idea de lo común antes de lo individual, lo cual es reflejo también del pensamiento indígena sobre la idea lo público, lo político y la política.

En la comunidad el sentido de lo público está relacionado con la pertenencia y la posesión, por lo que lo común no sólo es obligatorio, como las faenas, sino también es lo propio. El interés colectivo revestido por los procesos religiosos y la idea de la justicia son valores superiores que se materializan con el territorio, el cual a su vez proyecta las luchas por el poder político y las múltiples relaciones con el Estado. Éstas redefinen constantemente lo público en cuanto a que las organizaciones político-jurídicas de usos y costumbres van integrando en la agenda pública conquistas de orden social sobre cuestiones indígenas: el despojo y precarización del ámbito rural, la dinámica partidista y la extensión del modelo electoral que genera redes clientelares que afianzan el voto.

Para lo primero, el sistema de participación democrática contempla un modelo de gestión de lo indígena (la obligatoriedad de postulaciones indígenas a puestos de representación política). Para lo económico, las organizaciones con usos y costumbres no compatibilizan con las medidas de expansión de mercados. En el país los más profundos antagonismos entre lo privado y lo indígena son provocados por el choque entre sus visiones sobre el desarrollo (Ruvalcaba, 2004). En la comunidad el territorio es referencia de lo común, y lo común es el valor supremo que encauza los intereses colectivos (Guízar, 2005).

Dichos factores en la conformación de la agenda pública legitiman los efectos de esta fricción, identificada como antagonismo; coexistencia de lo diferente, o dramatismo entre formas diferentes de concebir lo político. Este proceso de legitimación trae consigo la formalización de los comportamientos al margen de los protocolos de relación entre lo indígena y el Estado. La formalización puede traducirse como institucionalización, en el sentido de que la democracia como forma de gobierno en el contexto neoliberal establece pautas en la relación de los agentes que intervienen en el ámbito político.

Podemos decir entonces, que la capacidad de las estructuras políticas indígenas para sortear el control político ejercido a partir de la transición hacia la democracia no fue una transformación pacífica, ni muchos menos consensuada. Los diferentes tratamientos hacia con las cuestiones indígenas que empezaron con el Cardenismo no trajeron como resultado la generación de capacidades para la organización política autónoma de los pueblos y comunidades con usos y costumbres. 
Esta configuración político-jurídica, en cambio, tuvo como consecuencia la sujeción por parte de los grupos indígenas a las instituciones del gobierno, resultado contrario a lo que se esperaba, dejando al margen la cuestión sobre la autonomía o la justicia social.

\section{CONCLUSIONES}

Finalmente, sostenemos que la construcción social de la justicia indígena en Ixmiquilpan es resultado de condiciones históricas particulares con efectos a largo plazo. El tratamiento de lo indígena en este sentido ha pasado por dos momentos importantes. El primero durante todo el proceso de Conquista, y el segundo con la política indigenista de Cárdenas y la subsecuente creación del Patrimonio Indígena del Valle del Mezquital, que no sólo propuso un sistema de incorporación económica del sector indígena, sino formó parte de un proyecto especial para introducirles al proceso e idea de la modernidad.

Actualmente impera un choque de visiones sobre el desarrollo político de los grupos indígenas. La ciudadanización, (así como la evangelización en la Conquista), ha dado paso a un proyecto que parece continuar con la idea de que, si bien se debe respetar a las organizaciones indígenas de usos y costumbres, en estas zonas se deben regenerar las condiciones para propiciar la mejora en la participación ciudadana en la toma de decisiones, y en general con todas las instituciones del Estado, siempre y cuando prime no sólo el respeto hacia ellas, sino también la aceptación de sus contenidos y formas de atención.

Este choque histórico de cosmovisiones, entre el proyecto propio de la sociedad y entre el proyecto de comunidad perteneciente a la cuestión indígena, pareciera alimentar la narrativa de desconfianza hacia el gobierno, pero principalmente de incomprensión hacia las formas diversas de comprender el mundo de lo político por parte del Estado, o "Estadocentrismo", con el cual las prácticas políticas e ideas de justicia propias de las estructuras políticas de las comunidades y pueblos indígenas han sobrepasado el ámbito estrictamente jurídico de la ciudadanía.

\section{REFERENCIAS BIBLIOGRÁFICAS}

Arbona, J. (2008). Ciudadanía política callejera: apropiación de espacios y construcción de horizontes políticos. En Ziccardi, A. (comp.). Procesos de urbanización de la pobreza y nuevas formas de exclusión social. Los retos de las políticas sociales de las ciudades latinoamericanas del siglo XXI. Buenos Aires: CLACSO-CROP.

Arias, A. (2005). Reforma indígena en México: dilemas y antinomias. Estudios Políticos, 5(8), pp. 57-89. https://doi.org/10.22201/fcpys.24484903e.2005.5.37655.

Barkin, D. y Lemus, B. (2015). Soluciones locales para la justicia ambiental. En Castro, F. y Hogenboom, B. (coords.). Gobernanza ambiental en América Latina. Buenos Aires: CLACSO.

Canabal, B. (2005). Actores rural-urbanos: proyectos e identidades. En Sánchez, H. (Coord.). Lo urbano-rural, ¿nuevas expresiones territoriales. Cuernavaca, Morelos: Universidad Nacional Autónoma de México, Centro Regional de Investigaciones Multidisciplinarias. 
Coase, R. (1937). The Nature of the Firm. Economica, 4(16), pp. 386-405. Recuperado de: http://www.jstor.org/stable/2626876.

DiMaggio, P. y Powell W. (1983). The iron cage revisted: Institutional isomorphism and collective rationality organizational fields. American Sociological Review, 48, pp. 147-160. https://doi.org/10.17323/1726-3247-2010-1-34-56.

Esposito, R. (2003). Communitas. Origen y destino de la comunidad. Buenos Aires: Amorrortu.

Garza, G. (2003). La urbanización de México en el siglo XX. México, D.F: Centro de Estudios Demográficos y de Desarrollo Urbano, El Colegio de México.

Guízar, F. (2005). Derecho, identidad, territorio y conflicto: propuesta metodológica para un estudio de caso. Desacatos, (17), pp. 127-152. https://doi.org/10.29340/ 17.1061.

Hernández, N. (2019). El enfoque sistémico en el institucionalismo histórico. Reflexión Política, 21(41), pp. 134-145. https://doi.org/10.29375/01240781.3233.

Instituto Nacional Electoral (2018). La agenda pendiente en materia de representación y participación política: voces de los pueblos y comunidades indígenas de México. México: INE.

López, H. (2013). De la gloria prehispánica al socialismo. Las políticas indigenistas del Cardenismo. Cuicuilco, (57), pp. 47-74. Recuperado de: http:/ /www.scielo.org.mx/ scielo.php?script $=$ sci_arttext\&pid $=$ S0185-16592013000200003\&lng $=\mathrm{es} \& \ln \mathrm{ln}=\mathrm{es}$.

López, L., Yocelevzky, R. y Zamora, G. (2019). Ciudadanías: desigualdad, exclusión e integración. Ciudad de México: Universidad Autónoma Metropolitana.

Mainwaring, S. y Welna, C. (eds.) (2003). Democratic Accountability en Latin América.

Oxford: Oxford University Press.

March, J. y Olsen, J. (1989). Rediscovering institutions: The organizational basis of politics. New York: Free Press.

Martínez, J. L. (2014). "Geoeducación y geocultura. Una aproximación a las articulaciones entre globalización, cultura y educación en el sistema-mundo”. Tesis de doctorado. Universidad Autónoma del Estado de Hidalgo, Hidalgo, México. Recuperado de: http://dgsa.uaeh.edu.mx:8080/bibliotecadigital/bitstream/handle/231104/1965/ Geoeducacion $\% 20 y \% 20$ Geocultura.pdf.

Méndez, M. J. (2005). Contradicción, complementariedad e hibridación en las relaciones entre lo rural y lo urbano. En Sánchez, H. (coord.). Lo urbano-rural, ¿nuevas expresiones territoriales? Cuernavaca, Morelos: Universidad Nacional Autónoma de México, Centro Regional de Investigaciones Multidisciplinarias.

Mendoza, S. (2007). Del gran hombre pequeño a los jefes. Tesis de doctorado. El Colegio de Michoacán, Michoacán, México. Recuperado de: https://colmich.repositorio institucional.mx/jspui/bitstream/1016/381/1/MendozaMendozaSilvia2007Tesis. pdf. 
North, D. (1995). Instituciones, cambio institucional y desempeño económico. México: Fondo de Cultura Económica.

O’Donnell, G. (2000). Teoría democrática y política comparada. Desarrollo Económico, 39(156), pp. 519-570. https://doi.org/10.2307/3455832.

Ostrom, E. (2000). El gobierno de los bienes comunes. México: Fondo de Cultura Económica.

Pérez, C. (2018). Expansión de la ciudad en la zona metropolitana de Pachuca: procesos desiguales y sujetos migrantes e inmobiliarios. Territorios, 38, pp. 41-65. https:// doi.org/10.12804/revistas.urosario.edu.co/territorios/a.5577.

Pierson, P. y Skocpol, T. (2008). El institucionalismo histórico en la ciencia política contemporánea. Revista Uruguaya de Ciencia Política, 17(1), pp. 7-38. Recuperado de: http:/ /www.redalyc.org/articulo.oa?id=297322673001.

Quezada, M. F. (2015). Las comunidades indígenas de Hidalgo. Ixmiquilpan Vol. III. Pachuca, Hidalgo: Universidad Autónoma del Estado de Hidalgo. Recuperado de: https://www.uaeh.edu.mx/investigacion/productos/6948/9_libro_las_comunida des_indigenas_de_hidalgo_ixmiquilpan_vol_iii.pdf.

- (2008). La migración hñãñú del Valle del Mezquital, Estado de Hidalgo. México: Comisión Nacional para el Desarrollo de los Pueblos Indígenas. Recuperado de: https://www.uaeh.edu.mx/investigacion/productos/5443/migracion_hnahnu_va lle_mezquital.pdf.

Ruvalcaba, J. (2004). Estado y violencia en el medio rural de México y Brasil. Desacatos, (14), pp. 169-186. https://doi.org/10.29340/14.1092.

Scott, J. (2004). Los dominados y el arte de la resistencia: discursos ocultos. México: Era.

Singer, M. (2016). Justicia electoral: México, participación y representación indígena. México: Tribunal Electoral del Poder Judicial de la Federación.

Tamayo, S. (2006). Espacios de ciudadanía, espacios de conflicto. Sociológica, 21(61), pp. 11-40. Recuperado de: http://www.redalyc.org/articulo.oa?id=305024682002.

Tönnies, F. (1979). Comunidad y sociedad. Barcelona: Ariel.

Velázquez, M. A. (2009). Comunidad y utopías en los movimientos indígenas y campesinos en Latinoamérica. En Clausen, H., et al (coords.). Utopías y globalización. Hermosillo, México: El Colegio de Sonora. 